\title{
BMJ Open Which parameters support disposition decision in suspected COVID-19 cases in the emergency department (ED): a German clinical cohort study
}

\author{
Martin Möckel (D) , ${ }^{1}$ Miriam Songa Stegemann, ${ }^{2}$ Volker Burst, ${ }^{3}$ Philipp Kümpers, ${ }^{4}$ \\ Joachim Risse, ${ }^{5}$ Felix Carlo Koehler, ${ }^{3}$ Domagoj Schunk, ${ }^{6}$ Jennifer Hitzek (i) ,1 \\ Tamara Elene Dietrich, ${ }^{1}$ Anna Slagman ${ }^{1}$
}

To cite: Möckel M, Stegemann MS, Burst V, et al. Which parameters support disposition decision in suspected COVID-19 cases in the emergency department (ED): a German clinical cohort study. BMJ Open 2021;11:e044853. doi:10.1136/ bmjopen-2020-044853

- Prepublication history and additional materials for this paper is available online. To view these files, please visit the journal online (http://dx.doi org/10.1136/bmjopen-2020044853).

Received 22 September 2020 Revised 23 February 2021 Accepted 12 March 2021

Check for updates

(C) Author(s) (or their employer(s)) 2021. Re-use permitted under CC BY-NC. No commercial re-use. See rights and permissions. Published by BMJ.

For numbered affiliations see end of article.

Correspondence to

Dr Martin Möckel;

martin.moeckel@charite.de

\section{ABSTRACT}

Objectives One major goal of the emergency department (ED) is to decide, whether patients need to be hospitalised or can be sent home safely. We aim at providing criteria for these decisions without knowing the SARS-CoV-2 test result in suspected cases.

Setting Tertiary emergency medicine.

Participants All patients were treated at the ED of the Charité during the pandemic peak and underwent SARSCoV-2 testing. Patients with positive test results were characterised in detail and underwent a 14-day-follow-up. Primary and secondary outcome measures Logistic regression and classification and regression tree (CART) analyses were performed to identify predictors (primary endpoint), which confirm safe discharge. The clinical endpoint was all-cause mortality or need for mechanical ventilation during index stay or after readmission. Results The primary test population of suspected COVID-19 consisted of $n=1255$ cases, $45.2 \%$ were women $(n=567)$. Of these, $n=110$ tested positive for SARS-CoV-2 (8.8\%). The median age of SARS-CoV-2-positive cases was 45 years (IQR: $33-66$ years), whereas the median age of the group tested negative for SARS-CoV-2 was 42 years (IQR: $30-60$ years) $(p=0.096) .43 .6 \%$ were directly admitted to hospital care.

CART analysis identified the variables oxygen saturation (<95\%), dyspnoea and history of cardiovascular (CV) disease to distinguish between high and low-risk groups. If all three variables were negative, most patients were discharged from $E D$, and the incidence of the clinical endpoint was $0 \%$. The validation cohort confirmed the safety of discharge using these variables and revealed an incidence of the clinical endpoint from $14.3 \%$ in patients with CV disease, $9.4 \%$ in patients with dyspnoea and $18.2 \%$ in patients with $0_{2}$ satuaration below $95 \%$. Conclusions Based on easily available variables like dyspnoea, oxygen saturation, history of CV disease, approximately $25 \%$ of patients subsequently confirmed with COVID-19 can be identified for safe discharge. Trial registration number DRKS00023117.

\section{INTRODUCTION}

The Coronavirus Disease 2019 (COVID-19) Pandemic poses unprecedented challenges
Strengths and limitations of this study

- This study constitutes a real-world cohort of patients tested positive for SARS-CoV-2 in the emergency department, and selection bias is, thus, very unlikely.

- All available clinical information were retrieved from patient files and, thus, a thorough analysis of clinical predictors of hospital admission was possible.

- The number of clinical endpoints like mechanical ventilation or death was low and, thus, the primary analysis of outcome prediction was not possible but described.

- Predictors of hospital admission were derived from a single-centre cohort, and thus generalisability might be questionable.

- The identified predictors for hospital admission were, however, validated in an independent, multicentre data set and results were similar, even though admission rates in general were higher in the multicentre cohort.

to modern healthcare worldwide. One of the particular challenges of COVID-19, the disease is caused by the most recently discovered coronavirus called SARS-CoV-2, is the possible presymptomatic or oligosymptomatic humanto-human transmission through small respiratory droplets through close-range contact ${ }^{1}$ explaining its exponential spread. Additionally, while most patients develop mild disease, severe courses ranging to critical disease with acute respiratory distress syndrome are particularly prevalent in patients with risk factors, that is, pre-existing pulmonary diseases; age over 60 years or obesity. ${ }^{2-7}$ Due to widespread transmission of the virus with localised clusters and outbreaks in many countries, the management of patients with severe disease stretched healthcare systems in several countries, to their limits, regionally and beyond.

Emergency departments (ED) typically are the first point of contact with the health 
system for patients with unclear and severe symptoms and disease states including COVID-19. ${ }^{8}$ On 1 March 2020, the first case in Berlin was diagnosed in our ED at Charité Universitätsmedizin Berlin located in the district Berlin Mitte. Charité university hospital as a tertiary care institution with 3000 beds is one of the largest university hospital centres in Europe. Compared with other European countries, Germany had the advantage of a longer period for health system contingency planning until more intense transmission occurred. This enabled healthcare providers to prepare for the pandemic by implementation of immediate-targeted action and a rapid, proactive and comprehensive approach. The early development and availability of diagnostic tests for virus detection by reverse transcription PCR (rt-PCR) from specimens, ${ }^{9}$ obtained from the upper respiratory tract (eg, oronasopharyngeal swabs), early establishment of testing capacities and the fact that the population initially affected in Germany was of younger age compared with other countries contributed to facilitating the country's response to the outbreak.

The public call for rapid sharing of research data resulted in a vast amount of high-ranking published papers from China and around the globe. Strategies for initial evaluation of suspected COVID-19 cases in the ED or fever outpatient clinic ${ }^{10}$ according to the original characteristics of the confirmed cases of COVID- $19^{11}$ were proposed. Modified clinical strategies were established in Germany. However, these proposals are based on clinical observations only and have not been backed up with data, yet.

For the present study, all patients tested for SARS-CoV-2 via rt-PCR from oronasopharyngeal swabs, obtained in the EDs of Charité Universitätsmedizin Berlin in the highly affected district Mitte, in the period from 1 March (case no. 1) to 15 April were analysed. The period covers the time of the first wave with the highest case numbers.

All patients with laboratory-confirmed SARS-CoV-2 infection were clinically characterised and followed up for at least 14 days. Factors associated with disposition and clinical outcome were determined and validated using independent multiple-centre cohort of confirmed cases of COVID-19 at the EDs of the University Hospital of Cologne, the University Hospital of Münster, the University Hospital of Kiel and the University Hospital of Essen, Germany between 1 March and 5 May 2020.

\section{METHODS}

\section{Derivation cohort}

ED patients at risk for infection were identified by a sitespecific algorithm and isolated prior to the initial assessment of the clinical status and, depending on the severity of the illness, were directed to appropriately equipped and separate treatment areas. Oronasopharyngeal swabs for the detection of SARS-CoV-2 RNA via rt PCR were performed in all suspected cases. Berlin's first COVID-19 case was confirmed on 1 March 2020 at the ED at Charité
Universitätsmedizin Berlin in the Mitte district in the EDs of Charité Campus Virchow Klinikum and Campus Charité Mitte. From 1 March to 15 April, all primary suspected cases of COVID-19 or cases in differential diagnostic clarification according to the definition published by the German Robert Koch Institute ${ }^{12}$ were included in the present study.

All following cases with confirmed SARS-CoV-2 infection were prospectively recorded and followed up in clinical routine. Hospital-wide and ED-specific protocols ${ }^{13}$ were established addressing establishment of a core team and key internal and external contact points, focusing on human, material and facility capacity, communication and data protection, training procedures, hand hygiene, personal protection equipment, waste management, triage, first contact and prioritisation, patient placement, moving of the patients in the facility and visitor access as well as environmental cleaning as proposed by international institutions.

Oligosymptomatic or asymptomatic self-presenter at our SARS-CoV-2 testing centre, which was set up as early as 3 March 2020, was not included in the analysis. Furthermore, confirmed cases tested positive prior to their presentation in the ED are not included in the series of primary suspected cases or cases in differential diagnostic clarification according to the definition published by the German Robert Koch Institute. ${ }^{12}$

In addition, a consecutive series of $\mathrm{n}=127$ SARS-CoV2-positive cases from the Registry for Clinical Presentation and Management of Patients With COVID-19 in the Emergency Room (ReCovER) and diagnosed in the ED of the University Hospitals of Cologne, Essen, Kiel and Münster between 1 March to 5 May 2020 were used for the validation of the multivariate model.

\section{Data collection and endpoints}

Clinical characteristics and in-hospital follow-up information of confirmed SARS-CoV-2 cases were extracted from electronic medical records. Particular attention was paid to vital parameters, certain chronic medical conditions, comorbidities and use of medication as ibuprofen, angiotensin converting enzyme inhibitors and angiotensin-II receptor antagonists.

Patients who did not meet the criteria for admission to the hospital and were discharged from ED were contacted by medical staff via telephone to inform them about their positive test result, educated on the importance of infection control and self-isolation and provided with instructions on the anticipated duration of isolation and warning signs that should prompt reevaluation. Another telehealth visit was scheduled within 14 days following the day of the ED presentation and confirmation of the infection at the University Hospitals of Berlin and Cologne.

The primary endpoint of the analyses was hospital admission after index presentation to the ED. Two further secondary combined clinical endpoints were used. The first clinical combined endpoint was intubation and death before discharge from the hospital or in case of initial 
outpatient treatment after readmission to hospital. The second clinical endpoint included the components of the first clinical endpoint (intubation and death) and additionally all intensive care unit (ICU) admissions during the index stay and all inpatient readmissions.

\section{Statistical analysis}

The present analysis focuses on confirmed SARS-CoV-2 cases. The population of patients with suspected COVID-19 infection also included the SARS-CoV-2 negative cases and was characterised in terms of age and sex in the derivation cohort. In the subgroup of positive SARS-CoV-2 cases, the primary endpoint was hospital admission and cases were compared between primary outpatients and admitted patients using descriptive analysis of characteristics, clinical parameters and postemergency care. With regard to the derivation of clinical decision strategies, clinical characteristics were analysed in consideration of the published proposals with regard to the primary endpoint. Descriptive analyses included the calculation of relative and absolute frequencies as well as median and IQR. Statistical differences were calculated using the $\mathrm{X}^{2}$ test for categorical variables and the Mann-Whitney test for continuous variables. A $p$ value of less than 0.05 was considered statistically significant. Due to the exploratory nature of the analysis, no corrections were made for multiple testing. Potential predictors for in-hospital treatment were first analysed univariately by above-mentioned statistical tests. All significant predictors were analysed by classification and regression analyses. ${ }^{14}$ For numeric variables, Youden optimised cut-off values were determined on basis of receiver operating characteristic curves. The identified predictors were then analysed as binary variables in logistic regression analysis and the best predicting variables were selected based on effect measure (OR) and model fit criteria (Cox \& Snell R-Square and Nagelkerkes R-Square) after selection of the first best prediction variable and cut-off. The above-mentioned procedures were then repeated at each step in the resulting subgroups. All identified predictors, including cut-offs and resulting subgroups, were graphically illustrated by classification and regression trees (CART). All the above-mentioned analyses were performed in the derivation cohort of Charité Universitätsmedizin Berlin.

\section{Validation cohort}

The final CART model, that is, the identified predictors and cut-offs, which was developed in the Charite-cohort and can thus be regarded as optimised for this cohort, was then applied to the validation cohort. This validation cohort consisted of patients enrolled into the ReCovER of the EDs of the University Hospital of Cologne, Münster, Essen and Kiel. The ReCovER registry has been approved by the Ethics Committee of the Medical Faculty of the University of Cologne (EK 20-1198, NCT04351854). The Essen and Münster cohorts of confirmed-patients with SARS-CoV-2 were enrolled with approval of the respective Ethics committees (file numbers: 20-9310-BO,
2929-571-b-S). The principles of the basic data protection regulation apply.

\section{Patient and public involvement statement}

The development of the research question, study design and outcome measures was developed by a team of experienced ED doctors and researchers who also concerned patients' perceived preferences and priorities. Patients were not involved directly in these processes. The results of this research work are going to be published open access and disseminated to interested patients via the website of the institution.

\section{RESULTS}

Figure 1 shows the patient flowchart of the Berlin cohort with the corresponding case numbers.

The primary population of suspected COVID-19 cases which received testing in the ED consisted of $n=1255$ cases, $45.2 \%$ were women $(n=567)$. The median age was 42 years (IQR: 31-60 years). The proportion of female patients was $39.1 \% \quad(n=23)$ in the group of confirmed SARS-CoV2 cases, which was slightly lower than the proportion of female patients who tested negative for SARS-CoV2 at 45.3\% ( $\mathrm{n}=502 ; \mathrm{p}=0.095)$. The median age of confirmed SARS-CoV-2 cases was in median 45 years (IQR: 33-66 years), whereas the median age of the group tested negative for SARS-CoV-2 was 42 years (IQR: 30-60 years) ( $\mathrm{p}=0.096$ ).

Figure 2 shows the daily test numbers during the study period and the proportion of positive cases that reached their maximum at the end of March. The online supplemental table 1S shows basic characteristics of the SARS-CoV-2 negatives $(\mathrm{n}=1070$, $\mathrm{nmiss}=38)$.

\section{Characteristics of patients with confirmed SARS-CoV-2 infection}

Table 1 depicts the clinical characteristics of patients with confirmed SARS-CoV-2 infection stratified by the primary endpoint (outpatient care or admission to the hospital). The proportion of women was higher in the outpatient group and the age was lower compared with patients admitted to the hospital on ED presentation. Significant differences in vital parameters were observed for temperature, respiratory rate and oxygen saturation. The frequency of diarrhoea, dyspnoea and abdominal pain was higher in hospitalised patients compared with outpatients. Among common risk factors, pre-existing cardiovascular (CV) and hepatic diseases significantly associated with in-patient treatment.

Laboratory parameters at admission were in general comparable and showed a broad overlap between outpatients and hospitalised patients. However, there were significant differences in $\mathrm{pCO}_{2}, \mathrm{pH}$, glucose, lactate, lymphocytes, lactate dehydrogenases (LDH), C reactive protein (CRP) and procalcitonin (table 2).

Prediction of hospitalisation in patients with SARS-CoV-2positive

Variables for classification and regression analyses were selected based on the bivariate association with 


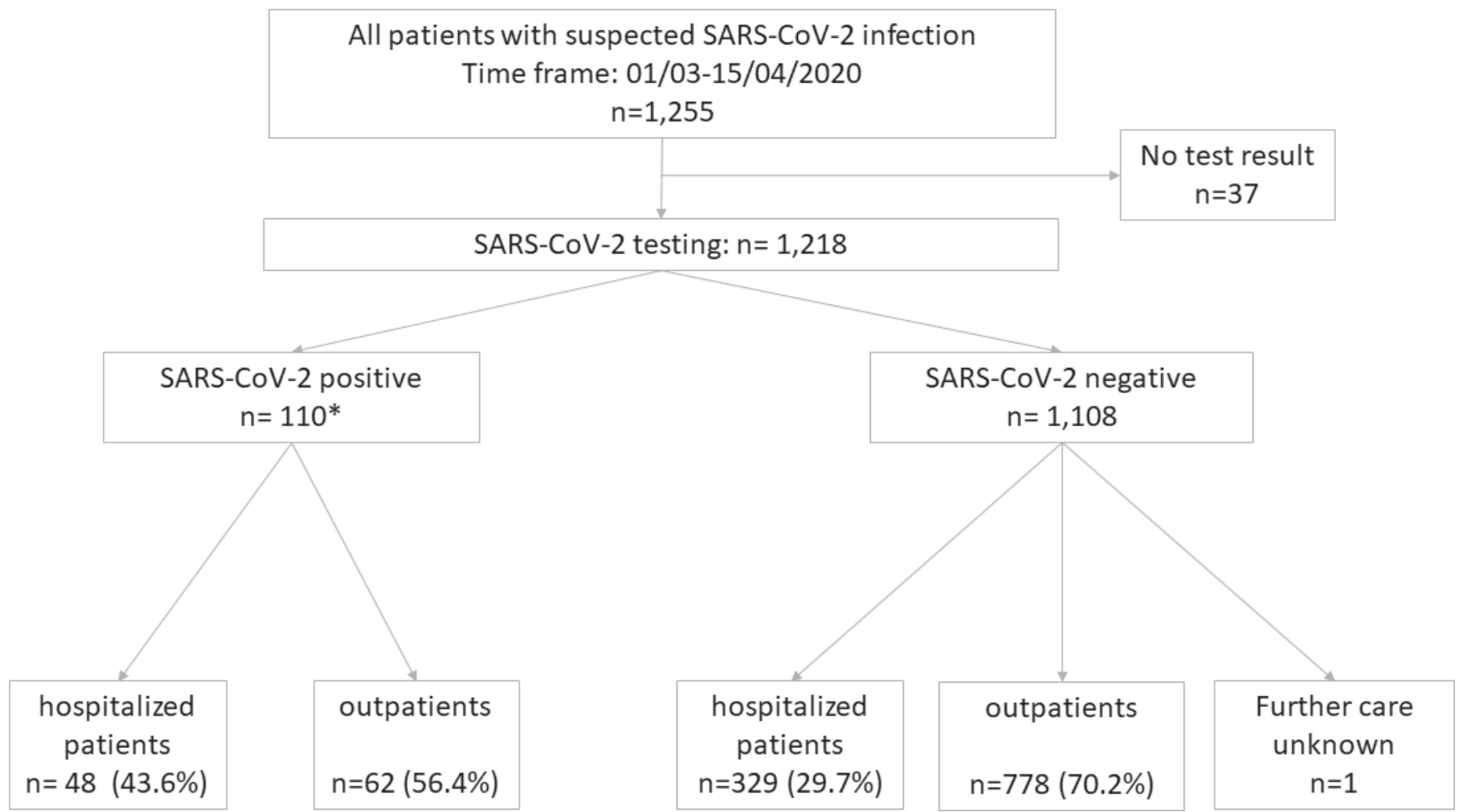

Figure 1 Patient flow diagram of the derivation cohort. *SARS-CoV-2 positive: $n=106$ positive tests performed at Charité laboratory, $n=4$ confirmed cases tested positive prior to their presentation in the ED (later confirmed in Charité laboratory) were also included in the analysis (total $n=110$ ). ED, emergency department.

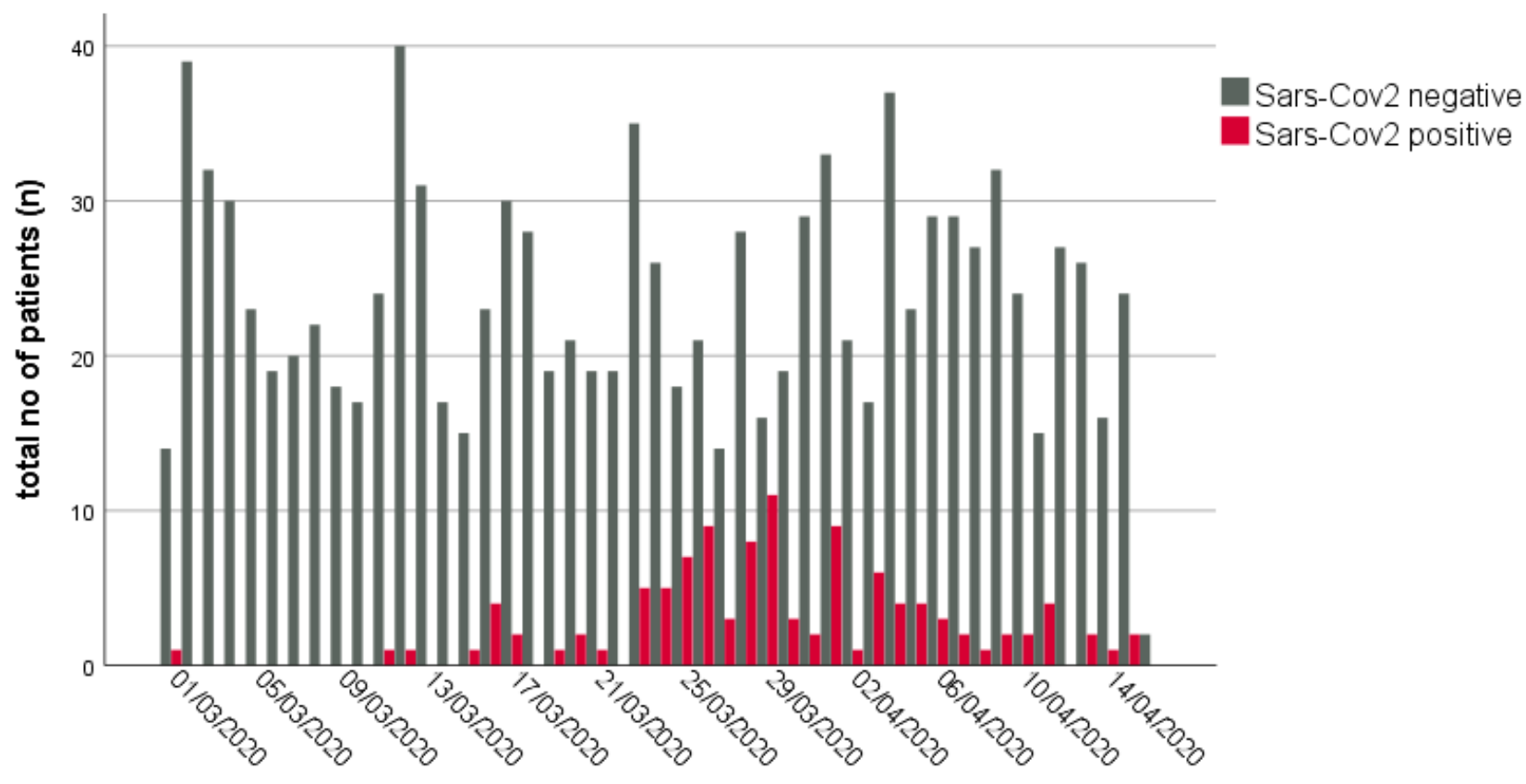

date of SARS-CoV2 test in the ED of the derivation cohort

Figure 2 Absolute number of SARS-CoV-2 negative tests (blue) and confirmed SARS-CoV-2 cases (red) in patients with ED at Charité Universitätsmedizin Berlin (CVK, CCM). CCM, CampusCharité Mitte; CVK, CampusVirchow Klinikum; ED, emergency department. 
Table 1 Demographic and clinical characteristics for patients with SARS-CoV2-positive with initial ambulatory treatment (outpatients) in the ED or inpatient treatment at Charite Universitätsmedizin Berlin

\begin{tabular}{|c|c|c|c|}
\hline & $\begin{array}{l}\text { SARS-CoV-2 positive hospitalised } \\
\text { patients }(n=48)\end{array}$ & $\begin{array}{l}\text { SARS-CoV-2 positive outpatient } \\
\text { treatment }(n=62)\end{array}$ & $P$ value \\
\hline Women \% (n) & $31.3(15)$ & $50.0(31)$ & 0.048 \\
\hline Age (median. IQR) & $56(42-78)$ & $38(30-49)$ & $<0.0001$ \\
\hline BMI (median. IQR) & $27(24-31)$ & $28(22-30)$ & 0.874 \\
\hline \multicolumn{4}{|l|}{ Vital signs (median. IQR) } \\
\hline BP syst. mm Hg & $135(118-150)$ & $133(122-147)$ & 0.912 \\
\hline BP diast. mm Hg & $75(69-86)$ & $80(74-90)$ & 0.061 \\
\hline Heart rate/min & 89 (79-99) & $90(80-101)$ & 0.705 \\
\hline Temperature ${ }^{\circ} \mathrm{C}$ & $37.8(37.2-38.8)$ & $37.3(36.6-37.8)$ & 0.004 \\
\hline Repspiratory rate/min & $18(16-24)$ & $16(15-18)$ & 0.009 \\
\hline Oxygen saturation \% & $95(93-97)$ & $99(97-100)$ & $<0.0001$ \\
\hline \multicolumn{4}{|l|}{ Vital signs at established risk cut-offs } \\
\hline BP syst. $<90 \mathrm{~mm} \mathrm{Hg} /$ diast. $\leq 60 \mathrm{~mm} \mathrm{Hg}$ & $10.4(5)$ & $12.9(8)$ & 0.012 \\
\hline Temperature $>37.3^{\circ} \mathrm{C}$ & $66.7(32)$ & $43.5(27)$ & 0.019 \\
\hline Respiratory rate $>18 / \mathrm{min}$ & $37.5(18)$ & $12.9(8)$ & 0.011 \\
\hline Respiratory rate $>30 / \mathrm{min}$ & $8.3(4)$ & 0 & 0.051 \\
\hline Oxygen saturation $<90 \%{ }^{*}$ & $12.5(6)$ & 0 & 0.009 \\
\hline \multicolumn{4}{|l|}{ Symptoms \% (n) } \\
\hline Fever & $75.0(36)$ & $62.9(39)$ & 0.137 \\
\hline Cough & $62.5(30)$ & $51.6(32)$ & 0.080 \\
\hline Haemoptysis & 0 & 0 & - \\
\hline Sore throat & $10.4(5)$ & $16.1(10)$ & 1.000 \\
\hline Rhinitis & $4.2(2)$ & $4.8(3)$ & 0.584 \\
\hline Headache/muscle pain & $25.0(12)$ & $46.8(29)$ & 0.707 \\
\hline Dyspnoea & $58.3(28)$ & $19.4(12)$ & $<0.0001$ \\
\hline Gl-symptoms & $12.5(6)$ & $6.5(4)$ & 0.064 \\
\hline Diarrhoea & $27.1(13)$ & $9.7(6)$ & 0.004 \\
\hline Nausea/emesis & $12.5(6)$ & $12.9(8)$ & 0.476 \\
\hline Loss of smell & $2.1(1)$ & $3.2(2)$ & 0.783 \\
\hline Abdominal pain & $10.4(5)$ & $3.2(2)$ & 0.029 \\
\hline Symptom onset time (days) & $7.5(5.0-10.8)$ & $3.5(2.0-6.0)$ & $<0.0001$ \\
\hline \multicolumn{4}{|l|}{ Risk factors \% (n) } \\
\hline Transplantation & $4.2(2)$ & $1.6(1)$ & 0.532 \\
\hline Tumour & $4.2(2)$ & $1.6(1)$ & 0.518 \\
\hline Cardiovascular disease & $52.1(25)$ & $14.5(9)$ & $<0.0001$ \\
\hline Respiratory disease & $20.8(10)$ & $16.1(10)$ & 0.747 \\
\hline Renal disease & $12.5(6)$ & $3.2(2)$ & 0.122 \\
\hline Hepatic disease & $14.6(7)$ & $1.6(1)$ & 0.022 \\
\hline Pregnancy & $4.2(2)$ & 0 & 0.290 \\
\hline
\end{tabular}

Demographic and clinical characteristics for patients with SARS-CoV2-positive with initial ambulatory treatment (outpatients) in the ED or inpatient treatment at Charité Universitätsmedizin Berlin (CVK, CCM). The cut-offs presented in this table are previously reported risk cut-offs and were not derived from the current data analysis.

${ }^{*}$ The cohort consists of patients with and without oxygen supplementation.

BMI, body mass index; BP, blood pressure; CCM, Campus Charité Mitte; CVK, Campus Virchow Klinikum; ED, emergency department; Gl, gastrointestinal.

the primary endpoint of hospital admission. Table 3 summarises the results of the best predictors in bivariate analyses. Decreased oxygen saturation, older age, presence of dyspnoea, longer duration of time since symptom onset, history of CV disease, elevated lactate, LDH and CRP were significantly associated with the 
Table 2 Laboratory parameters of patients with SARS-CoV2-positive with initial ambulatory treatment (outpatients) in the ED or inpatient treatment at Charité Universitätsmedizin Berlin (CVK, CCM)

\begin{tabular}{|c|c|c|c|}
\hline & $\begin{array}{l}\text { SARS-CoV-2 positive } \\
\text { hospitalised patients }(n=48)\end{array}$ & $\begin{array}{l}\text { SARS-CoV-2 positive } \\
\text { outpatient treatment }(n=62)\end{array}$ & \\
\hline $\mathrm{pO}_{2}(\mathrm{~mm} \mathrm{Hg})$ (median. IQR) (nmiss=38) & $28.5(20.4-34.8)$ & $29.2(23.5-36.2)$ & 0.605 \\
\hline $\mathrm{pCO}_{2}(\mathrm{~mm} \mathrm{Hg})($ median. IQR) (nmiss $=32)$ & $40.8(37.2-45.2)$ & $46.0(41.1-49.5)$ & 0.010 \\
\hline $\mathrm{pH}$ (median. IQR) (nmiss=32) & $7.41(7.38-7.45)$ & $7.39(7.36-7.41)$ & 0.017 \\
\hline $\mathrm{HCO}_{3}-(\mathrm{mmol} / \mathrm{L})($ median. IQR) (nmiss=32) & $26.0(24.1-27.3)$ & $26.9(24.9-28.4)$ & 0.132 \\
\hline BE (mmol) (median. IQR) (nmiss=35) & $1.20(-0.20-3.10)$ & $2.35(0.03-3.00$ & 0.483 \\
\hline Sodium (mmol/L) (median. IQR) (nmiss=32) & $137(134-140)$ & $138(136-141)$ & 0.144 \\
\hline Potassium (mmol/L) (median. IQR) (nmiss=31) & $4.0(3.7-4.2)$ & $4.0(3.7-4.3)$ & 0.684 \\
\hline Chloride (mmol/L) (median. IQR) (nmiss=33) & $103(101-107)$ & $104(101-107)$ & 0.447 \\
\hline Glucose (mg/dl) (median. IQR) (nmiss=33) & $130(110-150)$ & $108(97-123)$ & 0.001 \\
\hline Haemoglobin (g/L) (median. IQR) (nmiss=25) & $138(127-151)$ & $139(132-150)$ & 0.790 \\
\hline Lactate (mg/dL) (median. IQR) (nmiss=35) & $14.5(12.0-19.0)$ & $11.0(9.5-13.5)$ & $<0.0001$ \\
\hline WBC (10exp9/L) (median. IQR) (nmiss=26) & $6.9(5.1-8.9)$ & $5.7(4.5-8.4)$ & 0.406 \\
\hline Lymphocytes (\%) (median. IQR) (nmiss=54) & $0.92(0.66-1.36)$ & $1.41(1.19-2.07)$ & 0.003 \\
\hline CRP (mg/L) (median. IQR) (nmiss=31) & $60.6(27.1-118.8)$ & $14.4(3.9-30.4)$ & $<0.0001$ \\
\hline LDH (U/L) (median. IQR) (nmiss=44) & $394(291-501)$ & $246(212-326)$ & $<0.0001$ \\
\hline PCT ( $\mu \mathrm{g} / \mathrm{L})$ (median. IQR) (nmiss=51) & $0.11(0.07-1.55)$ & $0.05(0.04-0.08)$ & 0.002 \\
\hline
\end{tabular}

Laboratory parameters are shown as median and IQR for patients with SARS-CoV2-positive with initial ambulatory treatment (outpatients) in the ED or inpatient treatment at Charité Universitätsmedizin Berlin (CVK, CCM).

BE, base excess; CCM, Campus Charité Mitte; CRP, C reactive protein; CVK, Campus Virchow Klinikum; ED, emergency department; $\mathrm{HCO}_{3}$, bicarbonate; $\mathrm{LDH}$, lactate dehydrogenases; $\mathrm{pCO}_{2}$, partial pressure of carbon dioxide; $\mathrm{PCT}$, procalcitonin; $\mathrm{PH}$, power of hydrogen; $\mathrm{pO}_{2}$, partial pressure of oxygen; WBC, white cell count.

primary endpoint (admission to hospital). Oxygen saturation was the best predictor of hospital admission regarding area under the receiver operating characteristics curve $(0.822 ; 95 \%$ CI 0.735 to 0.909$)$ and model fit criteria ( $\mathrm{R}^{2}$ values: Cox and Snell $=0.237$; Nagelkerkes $=0.316$ ).

Table 3 Bivariate analysis of parameters regarding the prediction of hospital admission in patients with SARS-CoV2-positive in the emergency department (ED) at Charité Universitätsmedizin Berlin (CVK, CCM)

\begin{tabular}{|c|c|c|c|c|c|c|c|}
\hline & AUROC $(95 \% \mathrm{Cl})$ & $\begin{array}{l}P \text { value } \\
\text { ROC- } \\
\text { analysis }\end{array}$ & $\begin{array}{l}\text { Best cut- } \\
\text { off value }\end{array}$ & OR $(95 \% \mathrm{Cl})$ & $\begin{array}{l}\mathrm{P} \text { value } \\
\text { logistic } \\
\text { regression }\end{array}$ & $\begin{array}{l}\text { Cox and } \\
\text { Snell R- } \\
\text { square }\end{array}$ & $\begin{array}{l}\text { Nagel- } \\
\text { kerkes } \mathbf{R}^{2}\end{array}$ \\
\hline Oxygen saturation (\%) ${ }^{*}$ & $0.822(0.735$ to 0.909$)$ & $<0.0001$ & $95 \%$ & 36.6 (4.7 to 288.1$)$ & 0.001 & 0.237 & 0.316 \\
\hline Age (years) & 0.775 (0.680 to 0.869$)$ & $<0.0001$ & 55 & 9.5 (3.9 to 23.3 ) & $<0.0001$ & 0.225 & 0.302 \\
\hline Dyspnoea & NA & NA & NA & 7.2 (2.9 to 17.7$)$ & $<0.0001$ & 0.189 & 0.253 \\
\hline $\begin{array}{l}\text { Time since onset of } \\
\text { symptoms (days) }\end{array}$ & 0.751 (0.645 to 0.858 ) & $<0.0001$ & 6.5 & 6.0 (2.3 to 15.5$)$ & $<0.0001$ & 0.16 & 0.214 \\
\hline CV disease & NA & NA & NA & 5.6 (2.2 to 14.2$)$ & $<0.0001$ & 0.144 & 0.193 \\
\hline Lactate (mg/dL) & 0.745 (0.632 to 0.857 ) & $<0.0001$ & 12.5 & 2.2 (1.0 to 5.2$)$ & 0.062 & 0.033 & 0.044 \\
\hline LDH (U/L) & 0.798 (0.684 to 0.913$)$ & $<0.0001$ & 272 & 2.4 (0.9 to 6.9$)$ & 0.092 & 0.028 & 0.038 \\
\hline $\mathrm{CRP}(\mathrm{mg} / \mathrm{L})$ & 0.786 (0.682 to 0.890$)$ & $<0.0001$ & 30 & 2.0 (0.9 to 4.7$)$ & 0.099 & 0.026 & 0.035 \\
\hline
\end{tabular}

Results of the receiver operating characteristics analysis (ROC), best identified cut-off values (optimised by Youden-Index) according to the $\mathrm{ROC}$-curve and results of the univariate logistic regression analysis of dichotomised predictors. Also, goodness of fit criteria are reported $\left(R^{2}\right)$. The Cox and Snell $R^{2}$ is not standardised and can only be used to compare values between different models. The Nagelkerkes $R^{2}$ is standardised and produces values between 0 and 1 .

${ }^{*}$ The cohort consists of patients with and without oxygen supplementation.

AUROC, area under the receiver operating characteristics curve; CCM, Campus Charite Mitte; CRP, C reactive protein; CV, cardiovascular; CVK, Campus Virchow Klinikum; LDH, lactate dehydrogenase. 


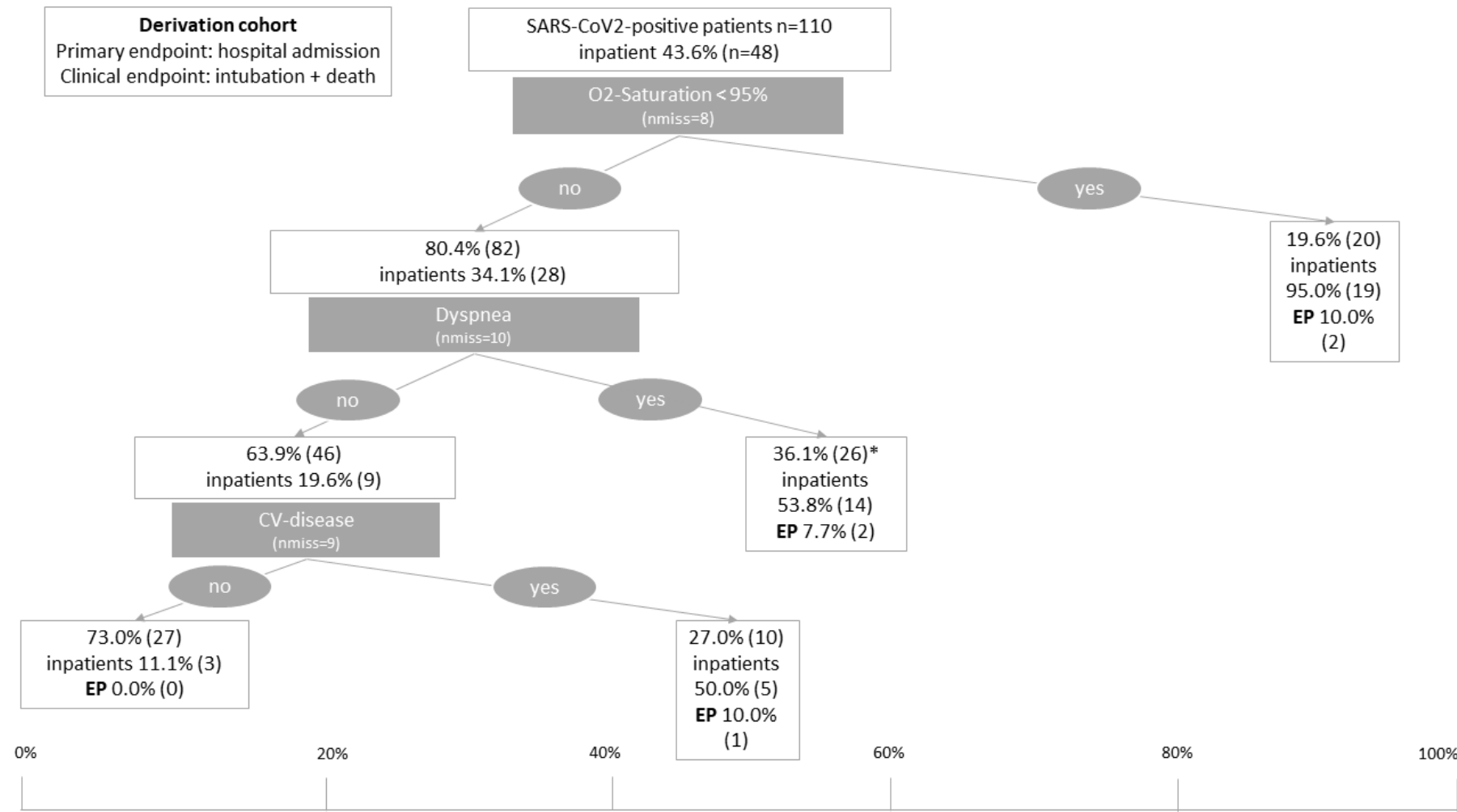

Figure 3 Classification and regression tree in the derivation cohort of Charité Universitätsmedizin Berlin. The position of the boxes on the x-axis illustrated the frequency of inpatient treatment in per cent while the size of the boxes is proportional to the size of the respective patient subgroup. Additionally to the proportion of patients who were admitted, the frequency of the clinical EP is displayed. *There was no further discriminating variable in the subgroup of patients with dyspnoea. CV, cardiovascular; EP, endpoint; nmiss, number of missing values; $\mathrm{O}_{2}$, oxygen.

Ninety-five per cent was the best cut-off point for oxygen saturation to discriminate between patients who were admitted to hospital and those who were discharged home. Decreased oxygen saturation below $95 \%$ was observed in $19.6 \%(n=20)$ of confirmed cases with SARS-CoV-2 infection. There was no further distinct discriminating variable in this subgroup with an already high hospitalisation rate of $95 \%(n=19)$ in the group of SARS-CoV-2 cases with oxygen saturation below $95 \%$. The clinical endpoint (intubation, death during index stay or after readmission) occurred in $10 \%(n=2)$ of this high-risk subgroup (figure 3$)$. Report of dyspnoea and history of CV disease were the best further discriminating variables within the subgroup of patients with an oxygen saturation at or above $95 \%(80.4 \% ; n=82)$. When the patient population was further divided into risk categories based on this information, patients with oxygen saturation at or above $95 \%$ but with reported dyspnoea or history of CV diseases showed a hospitalisation rate of $53.8 \%(n=14)$ and $50.0 \%(n=5)$, respectively. The risk for being intubated or death was $7.7 \%$ and $10 \%$, respectively. No clinical endpoint occurred in the remaining patients with lower risk for hospitalisation $(11.1 \%, \mathrm{n}=3)$ and none of the above-mentioned conditions $(n=27)$. The hospitalised patients with confirmed SARS-CoV-2 infection in the low risk group were diagnosed with cholangitis $(n=1)$ or viral pneumonia $(n=2)$ during their hospitalisation. All three hospitalised low-risk group patients were initially admitted to designated non-ICU COVID-19 wards. Both patients with viral pneumonia were transferred to ICU over the course of their stay. After recovery, all three patients were discharged home.

The derivation cohort consisted of $39.1 \%$ female patients and the median age was 45 years. The demographic characteristics of the validation cohorts were Cologne: female $47.5 \%$, median age 52 years; Essen: female $34.0 \%$, median age 71 years; Münster: female $25 \%$, median age 57 years; Kiel: female $41.7 \%$, median age 63 years. In the validation cohort, consisting of 127 confirmed cases with SARS-CoV-2 infection who presented to the EDs of the University Hospital of Cologne, Münster, Essen and Kiel, the proportion of inpatients was in general higher and, thus, also higher in the respective lower risk groups (figure 4$)$. In total, $66.9 \%(n=121)$ of SARS-CoV2-positive patients were admitted to hospital in the validation cohort. The proportion of patients with an oxygen saturation at or above $95 \%$ was higher as compared with the derivation cohort $(30.4 \%$; $=55)$ but resulted in a similar risk of admission to hospital $(94.5 \% ; n=52)$. The clinical endpoint occurred in $18.2 \%(\mathrm{n}=10)$ in this higher risk subgroup. The presence of symptoms of dyspnoea or preexisting cardiovascular disease resulted in risk groups with a proportion of inpatients of $68.8 \%$ and $81.0 \%$, respectively, and, thus, also higher as compared with the derivation cohort. Admission to hospital occurred in $63.2 \%$ in the low-risk group of the validation cohort (no dyspnoea and no CV disease). 


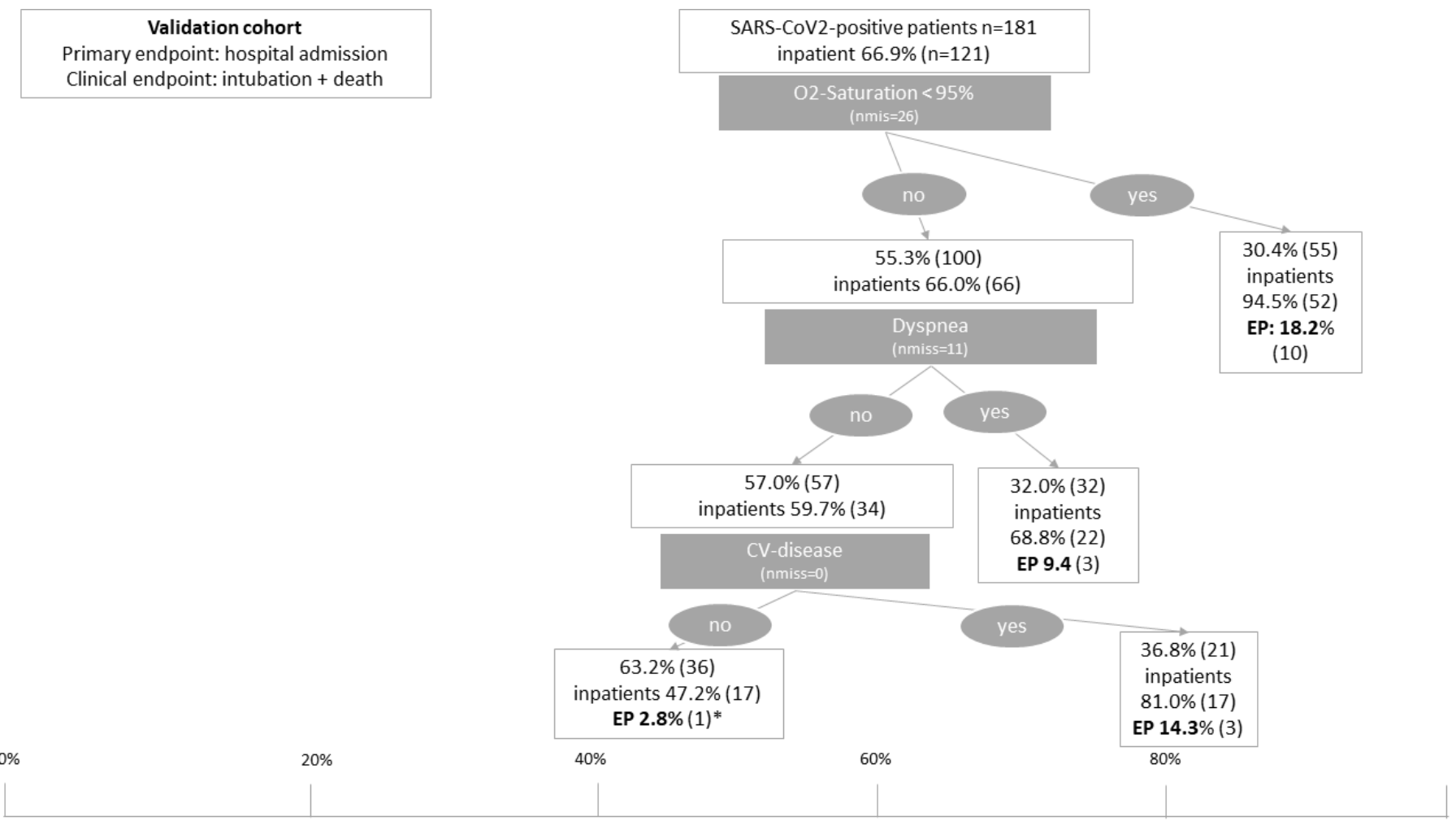

Figure 4 Classification and regression tree in the validation cohort of the EDs of the University Hospitals of Cologne, Münster, Essen and Kiel from the ReCovER registry. The position of the boxes on the x-axis illustrated the frequency of inpatient treatment in per cent while the size of the boxes is proportional to the size of the respective patient subgroup. In the low risk group, one patient had a clinical endpoint. This patient was admitted to the ward directly from the ED and had a terminal oncological disease, which led to patient's death without ICU admission. ED, emergency department; ICU, intensive care unit.

\section{Clinical endpoints in the derivation cohort}

The first combined clinical endpoint (intubation, death during index stay or after readmission) occurred in $5 \%$ $(n=5)$. The second combined clinical endpoint (intubation, death, inpatient readmission and all ICU admissions) was positive in $40 \%$ of all cases $(n=44)$. Of all admitted patients, $37.5 \%(\mathrm{n}=8)$ received oxygen supplementation. Of all 48 admitted patients, 23 patients were discharged during the follow-up period of 14 days. All other patients were still in hospital after 14 days and further length of hospital stay was not assessed. For these 23 patients, length of stay ranged between 1 and 12 days with a median length of stay (LOS) of 7 days (IQR: 4-10 days) (table 4).

\section{DISCUSSION}

In the present study, we provide evidence that the primary disposition of patients with suspected COVID-19 based on oxygen saturation, the cardinal symptom of dyspnoea and history of CV disease is safe and effective.

In contrast to most current strategies for risk stratification in COVID-19, which were developed focusing on severely ill hospitalised patients and the use of biomarkers like cardiac troponin and D-Dimer ${ }^{10}{ }^{11}{ }^{15-17}$, our study investigates a cohort of suspected cases of COVID-19 in the ED for the first time. Approximately, $10 \%$ of the suspected cases were confirmed with COVID-19\% and
43.6\% were primarily admitted to hospital care (see figure 1).

Using a CART-model, we were able to identify a risk stratification strategy for suspected cases of COVID-19 at initial presentation in the ED that is based on the clinical items oxygen saturation, dyspnoea and history of CV disease. Our risk stratification strategy uses easy-to-examine criteria and, thus, may reveal its distinctive strength, especially when healthcare systems are challenged: first, these criteria are explicitly defined and can readily be assessed at the time of the initial presentation due to suspected COVID-19. Second, these criteria rely on information taken exclusively from the initial patient history and their physical examination. This may facilitate risk assessment for physicians in the outpatient setting, where ordering laboratory tests may be too time-consuming, costly and difficult to perform during the current pandemic. Third, our prediction strategy may also help reduce uncertainty and result in evidence-based use of patient admission to inpatient care, which may be crucial when healthcare systems are being stretched.

There were differences in the proportion of patients admitted to hospital, with an in general higher hospitalisation rate in the validation cohort. This is most likely caused by different standards for hospital admission and availability of beds in the respective hospitals. In addition, it could be influenced by prehospital patient selection. 
Table 4 Clinical endpoints of patients with SARS-CoV2positive with initial ambulatory treatment (outpatients) in the ED or inpatient treatment at Charité Universitätsmedizin Berlin (CVK, CCM)

$\begin{array}{ll}\text { SARS-CoV- } & \text { SARS-CoV- } \\ \text { 2-positive } & \text { 2-positive } \\ \text { hospitalised } & \text { outpatient } \\ \text { patients }(n=48) & \text { treatment }(n=62)\end{array}$

\begin{tabular}{|c|c|c|}
\hline \multicolumn{3}{|c|}{ Primary hospitalised patients } \\
\hline $\begin{array}{l}\text { Intensive care unit } \\
\%(n)\end{array}$ & $60.4 \%(n=29)$ & 0 \\
\hline Intubation \% (n) & $10.4 \%(n=5)$ & 0 \\
\hline ECMO \% (n) & $0.0 \%(n=0)$ & 0 \\
\hline Death \% (n) ${ }^{*}$ & $4.2 \%(n=2)$ & 0 \\
\hline
\end{tabular}

Rehospitalisation of patients with primary outpatient treatment

\begin{tabular}{|c|c|c|}
\hline $\begin{array}{l}\text { Representation at } \\
\text { Charité ED \% (n) }\end{array}$ & DNA & $17.7 \%(n=11)$ \\
\hline $\begin{array}{l}\text { Hospital admission } \\
\text { Charité \% (n) }\end{array}$ & DNA & $6.5 \%(n=4)$ \\
\hline $\begin{array}{l}\text { ICU/intubation/ } \\
\text { ECMO \% (n) }\end{array}$ & DNA & $0.0 \%(n=0)$ \\
\hline $\begin{array}{l}\text { Discharged home } \\
\%(n)\end{array}$ & DNA & $3.2 \%(n=2) \dagger$ \\
\hline \multicolumn{3}{|c|}{$\begin{array}{l}\text { Telehealth follow-up of patients with outpatient treatment } \\
\text { during index stay }\end{array}$} \\
\hline $\begin{array}{l}\text { Re-presentation } \\
\text { other ED \% (n) }\end{array}$ & DNA & $4.8 \%(n=3)$ \\
\hline $\begin{array}{l}\text { Hospitalisation in } \\
\text { other hospital \% (n) }\end{array}$ & DNA & $3.2 \%(n=2)$ \\
\hline $\begin{array}{l}\text { ICU in other } \\
\text { hospital \% (n) }\end{array}$ & DNA & $0.0 \%(n=0)$ \\
\hline Death \% (n) & DNA & $0.0 \%(n=0)$ \\
\hline
\end{tabular}

Clinical endpoints of patients with SARS-CoV2-positive with initial ambulatory treatment (outpatients) in the ED or inpatient treatment at Charité Universitätsmedizin Berlin (CVK, CCM). Patients were followed up during their index stay and patients with primary ambulatory (outpatient) treatment received a follow-up call to assess further clinical course and endpoints.

*Patients who died were intubated before death. Thus the first clinical endpoint occurred in $n=5$ patients.

$\dagger \mathrm{t}=2$ of cases with primary outpatient treatment were readmitted to hospital and were still in hospital at follow-up.

CCM, Campus Charité Mitte; CVK, Campus Virchow Klinikum; DNA, does not apply; ECMO, extracorporeal membrane oxygenation; ED, emergency department; ICU, intensive care unit.

However, the risk stratification of the above-mentioned criteria (oxygen saturation, dyspnoea and CV diseases) could be confirmed in the validation cohort. Especially the identification of patients of high risk of hospital admission based on oxygen saturation at admission resulted in comparable risk of about $95 \%$. Of note, also other classification methods could have been used to identify predictors of hospital admission. The aim of the current analyses was to provide a robust risk classification algorithm, based on routinely available clinical information which could be easily implemented in clinical routine. CART analysis is a robust statistical method to detect reliable predictors. ${ }^{14}$

However, we must address several limitations of our study: our study was conducted and validated in the EDs of five German University Hospitals in total. This leads to a selection bias, since the majority of asymptomatic to very mild suspected cases of COVID-19 may have been seen in the outpatient setting in testing centres or by their general practitioner and not in the ED. Nevertheless, in the early phase ('first wave') of the pandemic, the university EDs took care of many mild cases also, as general care facilities were in a delayed building up process. Furthermore, patients defined as being at low risk due to our simplified risk stratification strategy may have other medical or psychosocial contraindications to be treated in outpatient setting in the current COVID-19 pandemic. For example, patients may have pre-existing disorders, such as relevant oncological or neuromuscular diseases, which are not part of our risk assessment strategy, but which may lead to an increased likelihood of progression to severe illness during the course of COVID-19. Moreover, oxygen saturation might be influenced by early, undocumented oxygen supplementation in the ED. This could have masked some more severe cases with falsely high oxygen saturation and thus an potential underestimation of the discriminatory abilities of oxygen saturation as a risk predictor for hospitalisation in the current study. Additionally, patients with severe impaired cognitive function, underlying psychiatric diseases or with little social support, may require inpatient care regardless of the severity of underlying COVID19. Furthermore, patients might be unable to adequately self-isolate themselves or are living in residential homes without adequate isolation management. In addition, our risk stratification does not apply to infants and children. The evaluation of infants and children needs to be developed separately. Another limitation is the inclusion of admitted patients only at the university hospitals of Essen, Kiel and Münster. Finally, during the pandemic, the ER utilisation was dramatically lower worldwide and it needs to be confirmed that the developed criteria are robust under regular circumstances. ${ }^{8}$

As a conclusion, we have developed an easy-to-determine risk stratification assay in a large set of suspected cases of COVID-19 that has been validated in an independent cohort from four separate German Emergency Medicine Centres. Our data provide preliminary evidence of a risk stratification strategy helping to determine whether hospital care is necessary in suspected cases of COVID-19. However, for a strong recommendation of our risk stratification and to confirm its safety and effectiveness, further trials comprising larger patient cohorts are warranted. For this purpose, we will make use of the already established ReCovER, an ongoing open retrospective survey platform facilitating anonymous data entry that is available on http://www.covid-em.org/. 


\section{Clinical significance}

Among all patients presenting with respiratory symptoms and suspicion of COVID-19, approximately $10 \%$ are tested positive for SARS-CoV-2.

More than 40\% of confirmed COVID-19 cases require hospital admission, mainly for symptomatic treatment and management of respiratory symptoms, hypoxia and other complications.

Factors for hospital admission are decreased oxygen saturation, dyspnoea and history of CV disease.

The primary disposition was confirmed safe by a very low event rate among those discharged home from the ED (0\% ICU admission as well as $0 \%$ mortality).

Future studies may develop additional criteria to increase the number of patients who can safely be treated as outpatients.

\section{Author affiliations}

${ }^{1}$ Emergency and Acute Medicine, Campus Mitte and Virchow, Charite Universitatsmedizin Berlin, Berlin, Germany

${ }^{2}$ Department of Infectious Diseases and Respiratory Medicine, Charite Universitatsmedizin Berlin, Berlin, Germany

${ }^{3}$ Department II of Internal Medicine: Nephrology, Rheumatology, Diabetes and General Internal Medicine, University Hospital Cologne, Cologne, Germany ${ }^{4}$ Emergency Medicine, Univerity Hospital Münster, Münster, Germany ${ }^{5}$ Emergency Medicine, Universitätsmedizin Essen, Essen, Germany ${ }^{6}$ Emergency and Acute Medicine, Universitätsklinikum Schleswig-Holstein Campus Kiel, Kiel, Germany

\section{Twitter Anna Slagman @annaSlagman}

Acknowledgements We acknowledge Clinical Research Unit (CRU) by Berlin Institute of Health (BIH), 10178 Berlin, Germany for providing support regarding the use of the data management software (RedCap).

Contributors AS and MM were involved in the conception and design of the study, the acquisition of the derivation data set, analysis and interpretation of data, drafted the manuscript and serve as guarantors for the manuscript. JH and MSS were involved in the conception and design of the study and JH and TED in the data acquisition of the derivation data set. VB, PK, JR, FCK and DS were involved in the acquisition and analysis of the validation data set. All authors were involved in the interpretation of data, critically revised the manuscript for important intellectual content, approved the final version to be published and agreed to be accountable for all aspects of the work. The corresponding author attests that all listed authors meet authorship criteria and that no others meeting the criteria have been omitted.

Funding The authors have not declared a specific grant for this research from any funding agency in the public, commercial or not-for-profit sectors.

Competing interests None declared.

Patient consent for publication Not required.

Ethics approval The Ethics Committee of the Charité-Universitätsmedizin Berlin approved the project as a substudy of an influenza study. (EA2/204/19).

Provenance and peer review Not commissioned; externally peer reviewed.

Data availability statement Data are available upon reasonable request. The data will be provided upon reasonable request from anna.slagman@charite.de.

Supplemental material This content has been supplied by the author(s). It has not been vetted by BMJ Publishing Group Limited (BMJ) and may not have been peer-reviewed. Any opinions or recommendations discussed are solely those of the author(s) and are not endorsed by BMJ. BMJ disclaims all liability and responsibility arising from any reliance placed on the content. Where the content includes any translated material, BMJ does not warrant the accuracy and reliability of the translations (including but not limited to local regulations, clinical guidelines, terminology, drug names and drug dosages), and is not responsible for any error and/or omissions arising from translation and adaptation or otherwise.

Open access This is an open access article distributed in accordance with the Creative Commons Attribution Non Commercial (CC BY-NC 4.0) license, which permits others to distribute, remix, adapt, build upon this work non-commercially, and license their derivative works on different terms, provided the original work is properly cited, appropriate credit is given, any changes made indicated, and the use is non-commercial. See: http://creativecommons.org/licenses/by-nc/4.0/.

ORCID iDs

Martin Möckel http://orcid.org/0000-0002-7691-3709

Jennifer Hitzek http://orcid.org/0000-0002-2213-5497

\section{REFERENCES}

1 He X, Lau EHY, Wu P, et al. Temporal dynamics in viral shedding and transmissibility of COVID-19. Nat Med 2020;26:672-5.

2 Dreher M, Kersten A, Bickenbach J. Charakteristik von 50 hospitalisierten COVID-19-patienten MIT und ohne ARDS. Dtsch Arztebl Int 2020;117:271-8.

3 Petrilli CM, Jones SA, Yang J, et al. Factors associated with hospital admission and critical illness among 5279 people with coronavirus disease 2019 in New York City: prospective cohort study. BMJ 2020;369:m1966.

4 CDC COVID-19 Response Team. Preliminary Estimates of the Prevalence of Selected Underlying Health Conditions Among Patients with Coronavirus Disease 2019 - United States, February 12-March 28, 2020. MMWR Morb Mortal Wkly Rep 2020;69:382-6.

5 Docherty AB, Harrison EM, Green CA, et al. Features of 20133 UK patients in hospital with covid-19 using the ISARIC WHO Clinical Characterisation Protocol: prospective observational cohort study. BMJ 2020;369:m1985

6 Richardson S, Hirsch JS, Narasimhan M, et al. Presenting characteristics, comorbidities, and outcomes among 5700 patients hospitalized with COVID-19 in the New York City Area. JAMA 2020;323:2052-9.

7 Lighter J, Phillips M, Hochman S, et al. Obesity in patients younger than 60 years is a risk factor for COVID-19 hospital admission. Clin Infect Dis 2020;71:896-7.

8 Slagman A, Behringer W, Greiner F, et al. Medical emergencies during the COVID-19 pandemic. Dtsch Arztebl Int 2020;117:545-52.

9 Corman VM, Landt O, Kaiser M, et al. Detection of 2019 novel coronavirus (2019-nCoV) by real-time RT-PCR. Eurosurveillance 2020;25.

10 Zhang J, Zhou L, Yang Y, et al. Therapeutic and triage strategies for 2019 novel coronavirus disease in fever clinics. Lancet Respir Med 2020;8:e11-12

11 Wang D, Hu B, Hu C, et al. Clinical characteristics of 138 hospitalized patients with 2019 novel coronavirus-infected pneumonia in Wuhan, China. JAMA 2020;323:1061.

12 Möckel M, Bachmann U, Behringer W, et al. How emergency departments prepare for virus disease outbreaks like COVID-19. Eur J Emerg Med 2020;27:161-2.

13 Control ECfDPa. Checklist for hospitals preparing for the reception and care of coronavirus 2019 (COVID-19) patients [online], 2020.

14 Muller R, Möckel M. Logistic regression and CART in the analysis of multimarker studies. Clinica Chimica Acta 2008;394:1-6.

15 Yang X, Yu Y, Xu J, et al. Clinical course and outcomes of critically ill patients with SARS-CoV-2 pneumonia in Wuhan, China: a singlecentered, retrospective, observational study. Lancet Respir Med 2020;8:475-81.

16 Chen N, Zhou M, Dong X, et al. Epidemiological and clinical characteristics of 99 cases of 2019 novel coronavirus pneumonia in Wuhan, China: a descriptive study. Lancet 2020;395:507-13.

17 Huang C, Wang Y, Li X, et al. Clinical features of patients infected with 2019 novel coronavirus in Wuhan, China. Lancet 2020;395:497-506 